\title{
On Forward Checking for Non-binary Constraint Satisfaction *
}

\author{
Christian Bessière ${ }^{1}$, Pedro Meseguer ${ }^{2}$, Eugene C. Freuder $^{3}$, and Javier Larrosa ${ }^{4}$ \\ 1 LIRMM-CNRS, 161 rue Ada, 34392 Montpellier, France \\ bessiere@lirmm.fr, \\ 2 IIIA-CSIC, Campus UAB, 08193 Bellaterra, Spain \\ pedro@iiia.csic.es, \\ 3 University of New Hampshire, Durham, NH 03824, USA \\ ecf@cs.unh.edu, \\ 4 Dep. LSI, UPC, Jordi Girona Salgado, 1-3, 08034 Barcelona, Spain \\ larrosa@lsi.upc.es
}

\begin{abstract}
Solving non-binary constraint satisfaction problems, a crucial challenge for the next years, can be tackled in two different ways: translating the non-binary problem into an equivalent binary one, or extending binary search algorithms to solve directly the original problem. The latter option raises some issues when we want to extend definitions written for the binary case. This paper focuses on the well-known forward checking algorithm, and shows that it can be generalized to several non-binary versions, all fitting its binary definition. The classical version, proposed by Van Hentenryck, is only one of these generalizations.
\end{abstract}

\section{Introduction}

Up to now, most of the research done in constraint satisfaction assumes that constraint problems can be exclusively formulated in terms of binary constraints. While many academic problems (n-queens, zebra, etc.) fit this condition, many real problems include non-binary constraints. It is well known the equivalence between binary and non-binary formulations [8]. Theoretically, this equivalence solves the issue of algorithms for non-binary problems. In practice, however, it presents serious drawbacks concerning spatial and temporal requirements, which often make it inapplicable. The translation process generates new variables, which may have very large domains, causing extra memory requirements for algorithms. In some cases, solving the binary formulation can be very inefficient [1]. In any case, this forced binarization generates unnatural formulations, which cause extra difficulties for constraint solver interfaces with human users.

An alternative approach consists in extending binary algorithms to nonbinary versions, able to solve non-binary problems in their original formulations.

* P. Meseguer and J. Larrosa were supported by an Integrated Action financed by the Generalitat de Catalunya, and by the Spanish CICYT project TIC96-0721-C02-02, C. Bessière was supported by an "action CNRS/NSF" under Grant no. 0690, and E.C. Freuder by the National Science Foundation under Grant No. IRI-9504316. 
This approach eliminates the translation process and its drawbacks, but it raises other issues, among which how a binary algorithm is generalized is a central one. For some algorithms, like backtracking or MAC, this extension presents no conceptual difficulties: their binary definitions allow only one possible nonbinary generalization. For other algorithms, like forward checking (FC), several generalizations are possible.

In this paper, we study how the popular FC algorithm can be extended to consider non-binary constraints. We present different generalizations, all collapsing to the standard version in the binary case. Our intention is mainly conceptual, trying to draw a clear picture of the different options for non-binary FC. We also provide some experimental results to initially assess the performance of the proposed algorithms with respect to other non-binary FC algorithms previously presented.

This paper is organized as follows. In Section 2, we present basic concepts used in the rest of the paper. In Section 3, we show the different ways in which binary FC can be generalized into non-binary versions. In Section 4, we provide properties and analysis of these generalizations, relating them to the algorithm FC + [1]. In Section 5, we provide experimental results of the proposed algorithms on random ternary problems. Finally, Section 6 contains some conclusions and directions for further research.

\section{Preliminaries}

A finite constraint network $\mathcal{C N}$ is defined as a set of $n$ variables $\mathcal{X}=\left\{x_{1}, \ldots, x_{n}\right\}$, a current domain $D\left(x_{i}\right)$ of possible values for each variable $x_{i}$, and a set $\mathcal{C}$ of constraints among variables. A constraint $c_{j}$ on the ordered set of variables $\operatorname{var}\left(c_{j}\right)=\left(x_{j_{1}}, \ldots, x_{j_{r(j)}}\right)$ specifies the relation $\operatorname{rel}\left(c_{j}\right)$ of the allowed combinations of values for the variables in $\operatorname{var}\left(c_{j}\right)$. $\operatorname{rel}\left(c_{j}\right)$ is a subset of $D_{0}\left(x_{j_{1}}\right) \times \cdots \times$ $D_{0}\left(x_{j_{r(j)}}\right)$, where $D_{0}\left(x_{i}\right)$ is the initial domain of $x_{i}$. (The definition of a constraint does not depend on the current domains.) An element of $D_{0}\left(x_{j_{1}}\right) \times \cdots \times D_{0}\left(x_{j_{r(j)}}\right)$ is called a tuple on $\operatorname{var}\left(c_{j}\right)$. An element of $D\left(x_{j_{1}}\right) \times \cdots \times D\left(x_{j_{r(j)}}\right)$ is called a valid tuple on $\operatorname{var}\left(c_{j}\right)$. We introduce the notions of initial and current domains to explicitly differentiate the initial network, $\mathcal{C} \mathcal{N}_{0}$, from a network $\mathcal{C N}$, obtained at a given node of a tree search after some operations (instantiations and/or filtering). The tuple $I_{P}$ on the ordered set of past variables $P$ represents the sequence of instantiations performed to reach a given node. The set $\mathcal{X} \backslash P$ of the future variables is denoted by $F$. The tuple $I_{P}$ on $P$ is said to be consistent iff for all $c$ such that $\operatorname{var}(c) \subseteq P, I_{P}$ satisfies $c$.

A value $a$ for variable $x$ is consistent with a constraint $c$ iff $x \notin \operatorname{var}(c)$, or there exists a valid tuple in $\operatorname{rel}(c)$ with value $a$ for $x$. A variable $x$ is consistent with a constraint $c$ iff $D(x)$ is not empty and all its values are consistent with $c$. A constraint $c$ is arc consistent iff for all $x \in \operatorname{var}(c), x$ is consistent with $c$. A set of constraints $C$ is arc consistent iff all its constraints are arc consistent [6, 7]. 
Let $C=\left\{c_{1}, \ldots, c_{k}\right\}$ be a set of constraints. We will denote by $A C(C)$ the procedure which enforces arc consistency on the set $C .{ }^{1}$ Given an arbitrary ordering of constraints $c_{1}, \ldots, c_{k}$, we say that $A C$ is applied on each constraint in one pass (denoted by $A C\left(\left\{c_{1}\right\}\right), \ldots, A C\left(\left\{c_{k}\right\}\right)$ ) when $A C$ is executed once on each individual constraint following the constraint ordering. We will denote by $\Pi_{x \in S} D(x)$ the Cartesian product of the domains of the variables in $S$. Let $\sigma$ be a tuple on the set of variables $S$. The projection of $\sigma$ on a subset $S^{\prime}$ of $S$, denoted by $\sigma\left[S^{\prime}\right]$, is the restriction of $\sigma$ to the variables of $S^{\prime}$. The projection $c\left[S^{\prime}\right]$ of the constraint $c$ on the subset $S^{\prime}$ of $\operatorname{var}(c)$ is a constraint defined by $\operatorname{var}\left(c\left[S^{\prime}\right]\right)=S^{\prime}$, and $\operatorname{rel}\left(c\left[S^{\prime}\right]\right)=\left\{t\left[S^{\prime}\right] / t \in \operatorname{rel}(c)\right\}$. The join of $\sigma$ and a relation $\operatorname{rel}(c)$ on $\operatorname{var}(c)$, denoted by $\sigma \bowtie \operatorname{rel}(c)$, is the set $\{t / t$ is a tuple on $S \cup \operatorname{var}(c)$, and $t[\operatorname{var}(c)] \in \operatorname{rel}(c)$, and $t[S]=\sigma\}$.

\section{From Binary to Non-binary FC}

FC (from now on, bFC) was defined in [4] for binary constraint networks. They described $\mathrm{bFC}$ as an algorithm pursuing this condition at each node,

there is no future unit having any of its labels inconsistent with any past unit-label pairs

where unit stands for variable, and label for value. Values in future domains are removed to achieve this condition, and if a future domain becomes empty, bFC backtracks. This condition is equivalent to require that the set $C_{p, f}^{b}$, composed of constraints connecting one past and one future variable, is arc consistent. To do this, it is enough performing arc consistency on the set $C_{c, f}^{b}$ of constraints involving the current and a future variable, each time a new current variable is assigned (Proposition 2, Section 4.1). In addition, arc consistency on this set can be achieved by computing arc consistency on each constraint in one single pass (Corollary 1, Section 4.1). With this strategy, after assigning the current variable we have,

$$
A C\left(C_{p, f}^{b}\right)=A C\left(C_{c, f}^{b}\right)=A C\left(\left\{c_{1}\right\}\right), \ldots, A C\left(\left\{c_{q}\right\}\right) \quad(\alpha)
$$

where $c_{i} \in C_{c, f}^{b}$ and $\left|C_{c, f}^{b}\right|=q$. So, bFC works as follows,

bFC: After assigning the current variable, apply arc consistency on each constraint of $C_{c, f}^{b}$ in one pass. If success (i.e., no empty domain detected), continue with a new variable, otherwise backtrack.

How can the FC strategy be extended for non-binary constraints? It seems reasonable to achieve arc consistency (the same level of consistency as bFC)

${ }^{1}$ Abusing notation, we will also denote by $A C(C)$ the set of values removed by the procedure $A C(C)$. 
on a set of constraints involving past and future variables. In the binary case, there is only one option for such a set: constraints connecting one past variable (the current variable) and one future variable. In the non-binary case, there are different alternatives. We analyze the following ones,

1. Constraints involving at least one past variable and at least one future variable;

2. Constraints or constraint projections involving at least one past variable and exactly one future variable;

3. Constraints involving at least one past variable and exactly one future variable.

Considering option (1), we define the set $C_{p, f}^{n}$ of the constraints involving at least one past variable and at least one future variable, and the set $C_{c, f}^{n}$ composed of constraints involving the current variable and at least one future variable. The big difference with the binary case is that, in these sets, we have to deal with partially instantiated constraints, with more than one uninstantiated variable. In this situation, the equivalences of $(\alpha)$ no longer hold for the non-binary case, that is,

$$
A C\left(C_{p, f}^{n}\right) \neq A C\left(C_{c, f}^{n}\right) \neq A C\left(\left\{c_{1}\right\}\right), \ldots, A C\left(\left\{c_{q}\right\}\right) \quad(\beta)
$$

where $c_{i} \in C_{c, f}^{n}$ and $\left|C_{c, f}^{n}\right|=q$. Then, we have different alternatives, depending on the set of constraints considered $\left(C_{p, f}^{n}\right.$ or $\left.C_{c, f}^{n}\right)$ and whether arc consistency is achieved on the whole set, or applied on each constraint one by one. They are the following,

nFC5: After assigning the current variable, make the set $C_{p, f}^{n}$ arc consistent. If success, continue with a new variable, otherwise backtrack.

nFC4: After assigning the current variable, apply arc consistency on each constraint of $C_{p, f}^{n}$ in one pass. If success, continue with a new variable, otherwise backtrack.

nFC3: After assigning the current variable, make the set $C_{c, f}^{n}$ arc consistent. If success, continue with a new variable, otherwise backtrack.

nFC2: After assigning the current variable, apply arc consistency on each constraint of $C_{c, f}^{n}$ in one pass. If success, continue with a new variable, otherwise backtrack.

Regarding options (2) and (3), we define the set $C_{p, 1}^{n}$ of the constraints involving at least one past variable and exactly one future variable, and the set $C_{c, 1}^{n}$ of the constraints involving the current variable and exactly one future variable. Analogously, we define the set $C P_{p, 1}^{n}$ of the constraint projections ${ }^{2}$ involving at least one past variable and exactly one future variable, and the set $C P_{c, 1}^{n}$ of the constraint projections involving the current variable and exactly one future variable. Both cases are concerned with the following generalization of $(\alpha)$ (proved in Section 4.1), stating that after assigning the current variable we have,

$$
A C\left(C_{p, 1}^{n}\right)=A C\left(C_{c, 1}^{n}\right)=A C\left(\left\{c_{1}\right\}\right), \ldots, A C\left(\left\{c_{q}\right\}\right)(\gamma)
$$

\footnotetext{
${ }^{2}$ A constraint projection is computed from the constraint definition which involves initial domains.
} 


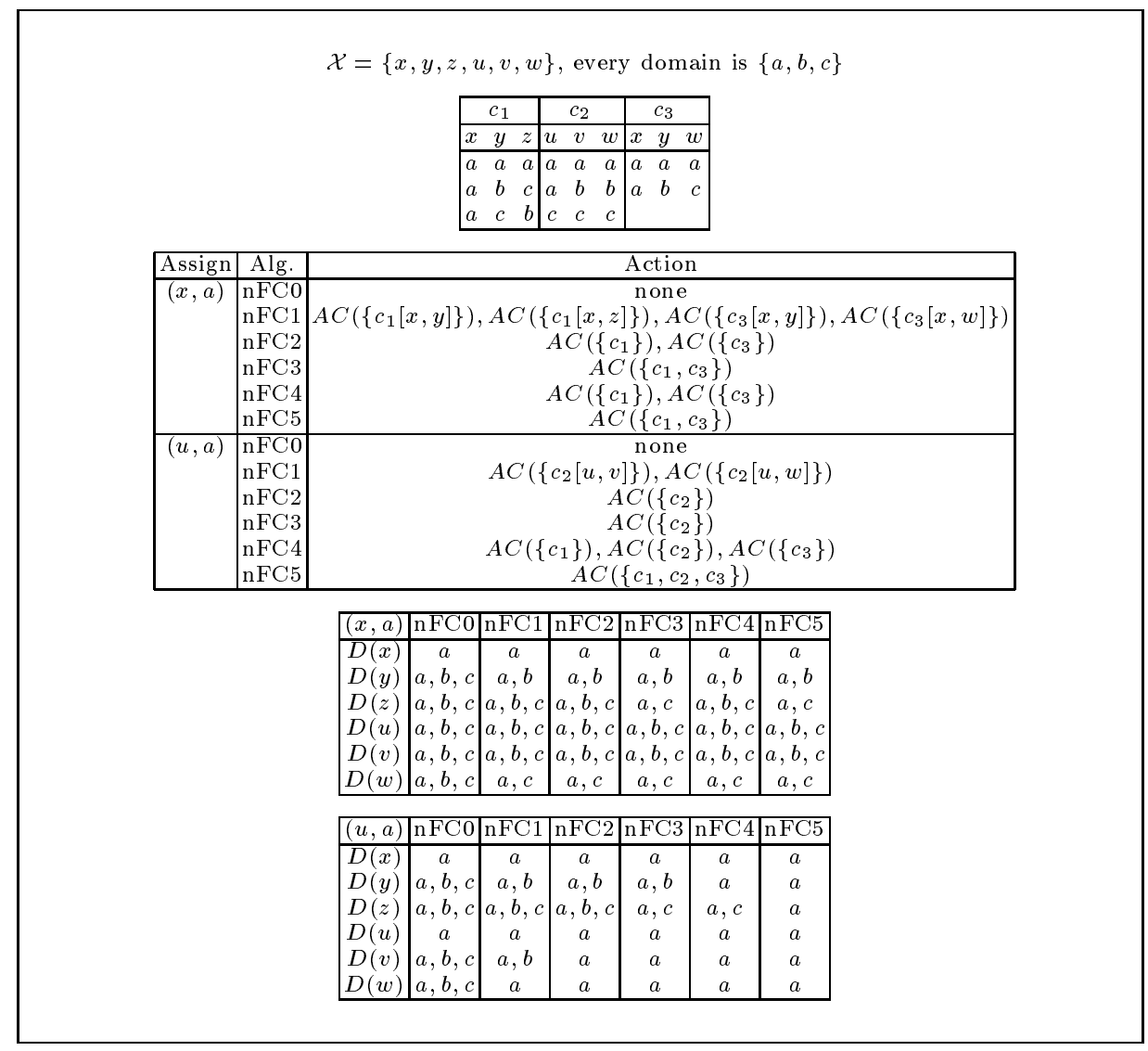

Fig. 1. A simple problem and the filtering caused by the six algorithms, after the assignments $(x, a)$ and $(u, a)$.

where $c_{i} \in C_{c, 1}^{n}$ and $\left|C_{c, 1}^{n}\right|=q$. As a result, only one alternative exists for each of the options (2) and (3), and they are the following,

nFC1: ([5]) After assigning the current variable, apply arc consistency on each constraint of $C_{c, 1}^{n} \cup C P_{c, 1}^{n}$ in one pass. If success, continue with a new variable, otherwise backtrack.

nFC0: ([10]) After assigning the current variable, apply arc consistency on each constraint of $C_{c, 1}^{n}$ in one pass. If success, continue with a new variable, otherwise backtrack.

To illustrate the differences between the six proposed algorithms, a simple example is presented in Figure 1. It is composed of 6 variables $\{x, y, z, u, v, w\}$, sharing the same domain $\{a, b, c\}$, and subject to three ternary constraints, $c_{1}(x, y, z), c_{2}(u, v, w)$ and $c_{3}(x, y, w)$. After the assignment $(x, a)$, none of the constraints have two instantiated variables. Therefore, $\mathrm{nFC} 0$ does no filtering. 
nFC1 applies arc consistency on the constraint projections of $c_{1}$ and $c_{3}$ on the subsets $\{x, y\},\{x, z\}$ and $\{x, w\}$, removing $c$ from $D(y)$ and $b$ from $D(w)$. nFC2 applies arc consistency on $c_{1}$ and later on $c_{3}$, pruning the same values as $\mathrm{nFC} 1$. Notice that if we consider these constraints in a different order, the filtering will be different. nFC3 achieves arc consistency on the subset $\left\{c_{1}, c_{3}\right\}$, which causes the filtering of nFC2 plus the removal of $b$ from $D(z)$. Given that $x$ is the first instantiated variable, $\mathrm{nFC} 4$ applies arc consistency on the same constraints as $\mathrm{nFC} 2$, and it causes the same filtering. For the same reason, nFC5 performs the same filtering as nFC3.

After the assignment $(u, a)$, none of the constraints have two instantiated variables. So, $\mathrm{nFC} 0$ does no filtering. $\mathrm{nFC} 1$ applies arc consistency on the constraint projections of $c_{1}$ on the subsets $\{u, v\}$ and $\{u, w\}$, removing $c$ from $D(v)$ and $c$ from $D(w)$. nFC2 applies arc consistency on $c_{2}$, and it removes $b$ and $c$ from $D(v)$ and $c$ from $D(w)$. nFC3 achieves arc consistency on the subset $\left\{c_{2}\right\}$, thus causing the same filtering as $\mathrm{nFC} 2$ (differences in $D(z)$ come from the previous assignment). $\mathrm{nFC} 4$ applies arc consistency on the constraints $c_{1}, c_{2}$ and $c_{3}$, removing $b$ from $D(y)$ and $D(z), b$ and $c$ from $D(v)$ and $c$ from $D(w)$. nFC5 achieves arc consistency on the whole constraint set. It removes $b$ from $D(y), c$ from $D(z), b$ and $c$ from $D(v)$ and $c$ from $D(w)$.

\section{Formal Results on nFC}

\subsection{Properties}

In the next results, we prove the equivalences of $(\gamma)$ used in Section 3 .

Proposition 1 Let $c$ be a constraint such that all its variables but one are instantiated. If $c$ is made arc consistent, it remains arc consistent after achieving arc consistency on any other problem constraint.

Proof. Let $\tilde{c}$ be another constraint sharing an uninstantiated variable $x_{j}$ with $c$. If $\tilde{c}$ is made arc consistent after $c$, this may cause further filtering in $D\left(x_{j}\right)$ but $c$ will remain arc consistent since all remaining values in $D\left(x_{j}\right)$ are already consistent with $c$.

Corollary 1 Let $C$ be a set of constraints such that all their variables but one are instantiated. Achieving arc consistency on $C$ is equivalent to make each of its constraints arc consistent in one pass.

Proposition 2 Let $P$ be the ordered set of past variables. Let $C_{p, 1}$ be the set of constraints involving at least one past variable and exactly one future variable. If each time a variable of $P$ was assigned, the set $C_{c, 1}$ of constraints involving that variable and one future variable was made arc consistent, the set $C_{p, 1}$ is arc consistent. 
Proof. Let us assume that $C_{c, 1}$ has been made arc consistent after assigning each variable in $P$. If $C_{p, 1}$ is not arc consistent, this means that there is at least one of its constraints $c$ which is not arc consistent. Let $x_{k}$ be the last assigned variable in $\operatorname{var}(c)$. Because of Proposition 1 this is in contradiction with the assumption that it was made arc consistent after assigning $x_{k}$. Therefore, $C_{p, 1}$ is arc consistent.

Regarding the correctness of the proposed algorithms, we have to show that they are sound (they find only solutions), complete (find all solutions) and terminate. All algorithms follow a depth-first strategy with chronological backtracking, so it is clear that all terminate. Then, we have to show soundness and completeness.

Proposition 3 Any $n F C i(i:\{0, \ldots, 5\})$ is correct.

Proof. Soundness. We prove that, after achieving the corresponding arc consistency condition, the tuple $I_{P}$ of past variables reached by any algorithm is consistent. When this tuple includes all variables, we have a solution. The sets of constraints to be made arc consistent by the proposed algorithms all include the set $C_{p, 1}$ of nFC0. By Proposition 1, we know that once those constraints are made arc consistent, they remain arc consistent after processing any other constraint. So, proving this result for $\mathrm{nFC} 0$ makes it valid for any $\mathrm{nFC} i$ algorithm $(i:\{0, \ldots, 5\})$. If $I_{P}$ of $\mathrm{nFC} 0$ is inconsistent then at least one constraint $c$ involving only variables in $P$ is inconsistent. Let $x_{i}$ and $x_{j}$ be the two last assigned variables in $\operatorname{var}(c)$, in this order. After assigning $x_{i}, c$ was in $C_{p, 1}$ which was made arc consistent. Assigning $x_{j}$ a value inconsistent with $c$ is in contradiction with the assumption that $C_{p, 1}$ was made arc consistent. So, $I_{P}$ is consistent.

Completeness. We show completeness for $\mathrm{nFC5}$, proofs for other algorithms are similar. Given a variable ordering, it is clear that $\mathrm{nFC5}$ visits all successors of nodes compatible with such ordering where the set $C_{p, f}^{n}$ can be made arc consistent. Let us suppose that there is a node solution, $I_{P}$, where all variables are past. If $x_{n}$ is the last variable to be instantiated, the parent node $I_{P \backslash\left\{x_{n}\right\}}$ is a node where $C_{p, f}^{n}$ can be made arc consistent. By induction, nFC5 visits the node solution $I_{P}$.

At a given node $k$, we define the filtering caused by an algorithm nFCi, $\Phi(n F C i, k)$, as the set of pairs $(x, a)$ where $a$ is a value removed from the future domain $D(x)$ by the corresponding arc consistency condition.

Proposition 4 At any node $k$ these relations hold,

1. $\Phi(n F C 0, k) \subseteq \Phi(n F C 1, k) \subseteq \Phi(n F C 2, k)$

2. $\Phi(n F C 2, k) \subseteq \Phi(n F C 3, k) \subseteq \Phi(n F C 5, k)$

3. $\Phi(n F C 2, k) \subseteq \Phi(n F C 4, k) \subseteq \Phi(n F C 5, k)$

Proof. Regarding $\mathrm{nFC} 0$ and $\mathrm{nFC1}$, the relation is a direct consequence of $C_{c, 1}^{n} \subseteq$ $C_{c, 1}^{n} \cup C P_{c, 1}^{n}$. Regarding $\mathrm{nFC1}$ and $\mathrm{nFC} 2$, constraint projections are semantically included in $C_{c, f}^{n}$. Regarding $\mathrm{nFC} 2$ and $\mathrm{nFC} 3$, applying arc consistency on each constraint of $C_{c, f}^{n}$ in one pass is part of the process of achieving arc consistency 
on the set $C_{c, f}^{n}$. Regarding $\mathrm{nFC} 3$ and $\mathrm{nFC} 5, C_{c, f}^{n} \subseteq C_{p, f}^{n}$. Regarding $\mathrm{nFC} 2$ and $\mathrm{nFC} 4, C_{c, f}^{n} \subseteq C_{p, f}^{n}$. Regarding $\mathrm{nFC} 4$ and $\mathrm{nFC}$, applying arc consistency on each constraint of $C_{p, f}^{n}$ in one pass is part of the process of achieving arc consistency on the set $C_{p, f}^{n}$.

Regarding $\mathrm{nFC} 3$ and $\mathrm{nFC} 4$, their filterings are incomparable as can be seen in example of Figure 1. (After assigning $(x, a), \mathrm{nFC} 3$ filtering is stronger than $\mathrm{nFC} 4$ filtering; the opposite occurs after assigning $(u, a)$.) A direct consequence of this result involves the set of nodes visited by each algorithm. Defining nodes $(n F C i)$ as the set of nodes visited by $\mathrm{nFC} i$ until finding a solution,

Corollary 2 Given a constraint network with a fixed variable and value ordering, the following relations hold,

1. $\operatorname{nodes}(n F C 2) \subseteq \operatorname{nodes}(n F C 1) \subseteq \operatorname{nodes}(n F C 0)$

2. $\operatorname{nodes}(n F C 5) \subset$ nodes $(n F C 3) \subset \operatorname{nodes}(n F C 2)$

3. nodes $(n F C 5) \subseteq \operatorname{nodes}(n F C 4) \subseteq \operatorname{nodes}(n F C 2)$

\subsection{Complexity Analysis}

In this subsection, we give upper bounds to the number of constraint checks the different $\mathrm{nFC}$ algorithms perform at one node. First, let us give an upper bound to the number of checks needed to make a variable $x_{j}$ consistent with a given constraint $c$. For each value $b$ in $D\left(x_{j}\right)$, we have to find a subtuple $\sigma$ in $\Pi_{x \in \operatorname{var}(c) \backslash\left\{x_{j}\right\}} D(x)$ such that $\sigma$ extended to $\left(x_{j}, b\right)$ is allowed by $c$. So, the number of checks needed to make $x_{j}$ consistent with $c$ is in $O(d \cdot|V|)$, where $V=\Pi_{x \in \operatorname{var}(c) \backslash\left\{x_{j}\right\}} D(x)$, and $d$ denotes the maximal size of a domain.

In $\mathrm{nFC0}$, a constraint $c$ is made arc consistent at a given node iff $\operatorname{var}(c)$ contains only one future variable. Thus, enforcing arc consistency on $c$ is in $O(d)$ since $|V|=1$. (Domains of past variables are singletons.) Therefore, the number of checks performed by $\mathrm{nFC} 0$ at one node is in $O\left(\left|C_{c, 1}^{n}\right| \cdot d\right)$. For the same reason the number of checks performed by $\mathrm{nFC1}$ at one node is in $O\left(\left|C_{c, 1}^{n} \cup C P_{c, 1}^{n}\right| \cdot d\right)$, assuming that the constraint projections have been built in a preprocessing phase.

In $\mathrm{nFC} 2$ and $\mathrm{nFC} 4,|V|$ is bounded above by $d^{|\operatorname{var}(c) \cap F|-1}$ for a given constraint $c$, and a given future variable $x_{j}$ in $\operatorname{var}(c)$. Thus, making $x_{j}$ consistent with $c$ is bounded above by $d \cdot d^{|\operatorname{var}(c) \cap F|-1}$, and enforcing arc consistency on $c$ is in $O\left(|\operatorname{var}(c) \cap F| \cdot d^{|\operatorname{var}(c) \cap F|}\right)$ since there are $|\operatorname{var}(c) \cap F|$ variables to make arc consistent with $c$. So, the number of checks performed at one node is in $O\left(\left|C_{c, f}^{n}\right|\right.$. $\left.|\operatorname{var}(c) \cap F| \cdot d^{|\operatorname{var}(c) \cap F|}\right)$ for $\mathrm{nFC} 2$, and in $O\left(\left|C_{p, f}^{n}\right| \cdot|\operatorname{var}(c) \cap F| \cdot d^{|\operatorname{var}(c) \cap F|}\right)$ for $\mathrm{nFC} 4$.

At a given node in the search, $\mathrm{nFC} 3$ (resp. $\mathrm{nFC5}$ ) deals with the same set of constraints as $\mathrm{nFC2}$ (resp. $\mathrm{nFC} 4$ ). The difference comes from the propagations nFC3 (resp. nFC5) performs in order to reach an arc consistent state on $C_{c, f}^{n}$ (resp. $C_{p, f}^{n}$ ), whereas $\mathrm{nFC} 2$ (resp. nFC4) performs "one pass" arc consistency on them. Thus, if we suppose that arc consistency is achieved by an optimal algorithm, such as GAC4 [7] or GAC-schema [2], the upper bound in the number of constraint checks performed by $\mathrm{nFC} 3$ (resp. nFC5) at a given node is the same 
as nFC2 (resp. nFC4) bound. (With an AC3-like algorithm [6], nFC3 and nFC5 have a greater upper bound.)

\section{3 $\quad \mathrm{FC}+$ and $\mathrm{nFC1}$}

The hidden variable representation is a general method for converting a nonbinary constraint network into an equivalent binary one $[3,8]$. In this representation, the problem has two sets of variables: the set of the ordinary variables, those of the original non-binary network, with their original domain of values, plus a set of hidden variables, or h-variables. There is a h-variable $h_{c}$ for each constraint $c$ of the original network, with $\operatorname{rel}(c)$ as initial domain (i.e., the tuples allowed by $c$ become the values in $D_{0}\left(h_{c}\right)$ ). A h-variable $h_{c}$ is involved in a binary constraint with each of the ordinary variables $x$ in $\operatorname{var}(c)$. Such a constraint allows the set of pairs $\left\{(a, t) / a \in D_{0}(x), t \in D_{0}\left(h_{c}\right), t[x]=a\right\}$.

$\mathrm{FC}+$ is an algorithm designed to run on the hidden representation [1]. It operates like bFC except that when the domain of a h-variable is pruned, $\mathrm{FC}+$ removes from adjacent ordinary variables those values whose support has been lost. Besides, FC + never instantiates h-variables. When all its neighboring (ordinary) variables are instantiated, the domain of a h-variable is already reduced to one value. Its assignment is, in a way, implicit. Therefore, there is a direct correspondence between the search space of $\mathrm{FC}+$ and any nFC. The following proposition shows that $\mathrm{FC}+$ is equivalent to $\mathrm{nFC1}$.

Proposition 5 Given any non-binary constraint network, nFC1 visits exactly the same nodes as $F C+$ applied to the hidden representation, provided that both algorithms use the same variable and value orderings.

Proof. Because of the algorithmic description of $\mathrm{FC}+$, we know that h-variables may only have their domain pruned by the bFC look ahead. An arbitrary hdomain, $D\left(h_{c}\right)$, may only be pruned if $P \cap \operatorname{var}(c) \neq \emptyset$, and $D\left(h_{c}\right)=\left\{\left(I_{P} \bowtie\right.\right.$ $\operatorname{rel}(c))[\operatorname{var}(c)]\}$. Domains of ordinary variables may only be pruned by the extra look ahead of $\mathrm{FC}+$. At a given node, value $b$ for a future variable $x_{j}$ belongs to $D\left(x_{j}\right)$ iff it still has support from all its adjacent h-variables that may have been pruned. That is, $\forall x_{j} \in F, b \in D\left(x_{j}\right)$ iff $\forall c$ s.t. $\operatorname{var}(c) \cap P \neq \emptyset$ and $x_{j} \in \operatorname{var}(c)$, $b \in D\left(h_{c}\right)\left[x_{j}\right]=\left(\left(I_{P} \bowtie \operatorname{rel}(c)\right)[\operatorname{var}(c)]\right)\left[x_{j}\right]=\left(I_{P} \bowtie \operatorname{rel}(c)\right)\left[x_{j}\right]$. Now, because of its definition, we know that at a given node nFC1 ensures that value $b$ for a future variable $x_{j}$ belongs to $D\left(x_{j}\right)$ iff it is consistent with the projections $\operatorname{rel}(c)\left[\operatorname{var}(c) \cap P \cup\left\{x_{j}\right\}\right]$ for all the constraints $c$ such that $\operatorname{var}(c) \cap P \neq \emptyset$ and $x_{j} \in \operatorname{var}(c)$. That is, $\forall x_{j} \in F, b \in D\left(x_{j}\right)$ iff $\forall c$ s.t. $\operatorname{var}(c) \cap P \neq \emptyset$ and $x_{j} \in \operatorname{var}(c), b \in\left(I_{P} \bowtie\left(\operatorname{rel}(c)\left[\operatorname{var}(c) \cap P \cup\left\{x_{j}\right\}\right]\right)\right)\left[x_{j}\right]=\left(I_{P} \bowtie \operatorname{rel}(c)\right)[\operatorname{var}(c) \cap$ $\left.P \cup\left\{x_{j}\right\}\right]\left[x_{j}\right]=\left(I_{P} \bowtie \operatorname{rel}(c)\right)\left[x_{j}\right]$, which is exactly what we found for $\mathrm{FC}+$. Since $\mathrm{FC}+$ and $\mathrm{nFC} 1$ prune exactly the same values on the ordinary variables at a given node, we have the proof. 


\section{$5 \quad$ Experimental Results}

We have performed some experiments to preliminary assess the potential of the proposed algorithms. In our experiments we have used random problems extending the well known four-parameter binary model [9] to ternary problems as follows. A ternary random problem is defined by four parameters $\left\langle n, m, p_{1}, p_{2}\right\rangle$ where $n$ is the number of variables, $m$ is the cardinality of their domains, $p_{1}$ is the problem connectivity as the ratio between existing constraints and the maximum number of possible constraints (the problem has exactly $p_{1} n(n-1)(n-2) / 6$ constraints), and $p_{2}$ is the constraint tightness as the proportion of forbidden value triplets between three constrained variables (the number of forbidden value triplets is exactly $T=p_{2} m^{3}$ ). The constrained variables and their nogoods are randomly selected following a uniform distribution.

We performed experiments on the following classes of problems:

(a) $\left\langle 10,10,100 / 120, p_{2}\right\rangle$,

(b) $\left\langle 30,6,75 / 4060, p_{2}\right\rangle$,

(c) $\left\langle 75,5,120 / 67525, p_{2}\right\rangle$.

Regarding connectivity, (a) is a dense class while (b) and (c) are sparse classes. The complexity peak location appears in (a) at low tightness, in (b) at medium tightness, and in (c) at high tightness.

We solved 50 instances for each set of parameters, using $\mathrm{nFC} 0, \mathrm{nFC1}, \mathrm{FC}+$, $\mathrm{nFC} 2, \mathrm{nFC} 3, \mathrm{nFC} 4$, and $\mathrm{nFC} 5,{ }^{3}$ with the heuristic minimum $\frac{\text { domain size }}{\text { degree }}$ for variable selection and lexicographic value selection. Figure 2 shows the mean number of visited nodes to solve each problem class. Only the complexity peak region is shown. With no surprise, it is in agreement with Corollary 2, which establishes that $\mathrm{nFC0}$ is the algorithm visiting the most nodes while $\mathrm{nFC} 5$ is the one that visits the least nodes. Because of Proposition $5, \mathrm{nFC} 1$ and $\mathrm{FC}+$ visit the same nodes. The new information is about the relation between $\mathrm{nFC} 3$ and nFC4, algorithms unordered by Corollary 2 . Consistently in the three problem classes, $\mathrm{nFC} 4$ visits less nodes than $\mathrm{nFC} 3$, which implies that $\mathrm{nFC} 4$ performs more pruning than $\mathrm{nFC} 3$.

Figures 3 and 4 show the average computational effort ${ }^{4}$ (as mean number of consistency checks and mean CPU time) required. We observe that, for easy problems (with peak at low tightness) the winner is $\mathrm{nFC0}$, the algorithm that performs the simplest lookahead. For this class of problems, sophisticated forms of lookahead do not pay-off: the proposed algorithms nFC1 to nFC5 are 1.8 to 4.8 times slower than $\mathrm{nFC} 0$ at the peak. $\mathrm{FC}+$ on the hidden representation is orders of magnitude slower. For problems with the peak at medium tightness, no single algorithm clearly outperforms the others. $\mathrm{nFC} 0, \mathrm{nFC} 1, \mathrm{nFC} 2$, and $\mathrm{nFC} 5$ are very close. $\mathrm{nFC} 3$ and $\mathrm{nFC} 4$ are slightly worse. The bad behavior of $\mathrm{FC}+$ is confirmed. For difficult problems with the peak located at high tightness, the proposed

\footnotetext{
${ }^{3}$ In nFC3 and nFC5, the technique used to achieve arc consistency on a set of constraints is a brute force non optimal GAC3-based algorithm.

${ }^{4}$ This effort includes the preprocessing phase for $n F C 1$ and the conversion into the hidden representation for $\mathrm{FC}+$.
} 

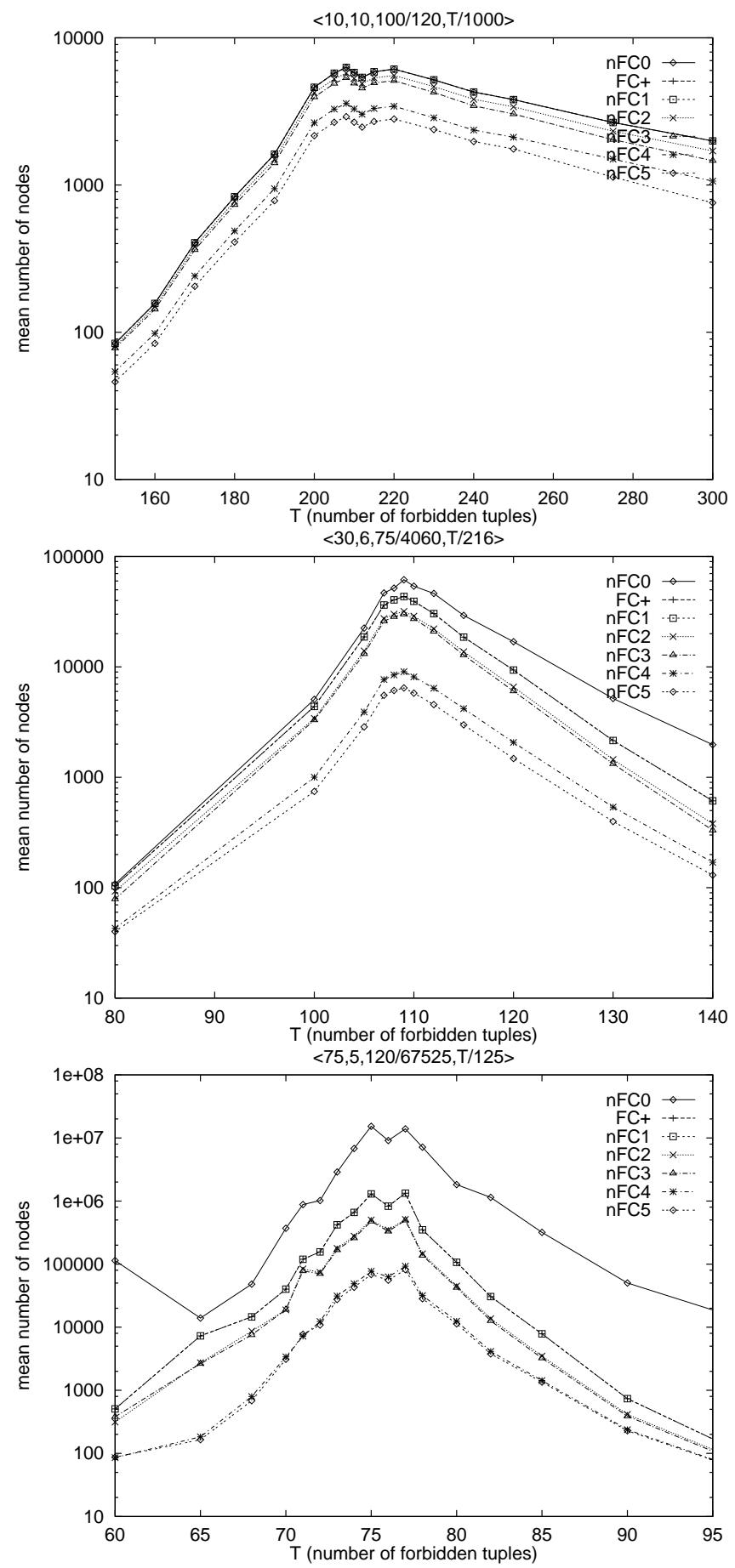

Fig. 2. Average number of visited nodes for three classes of ternary random problems. 

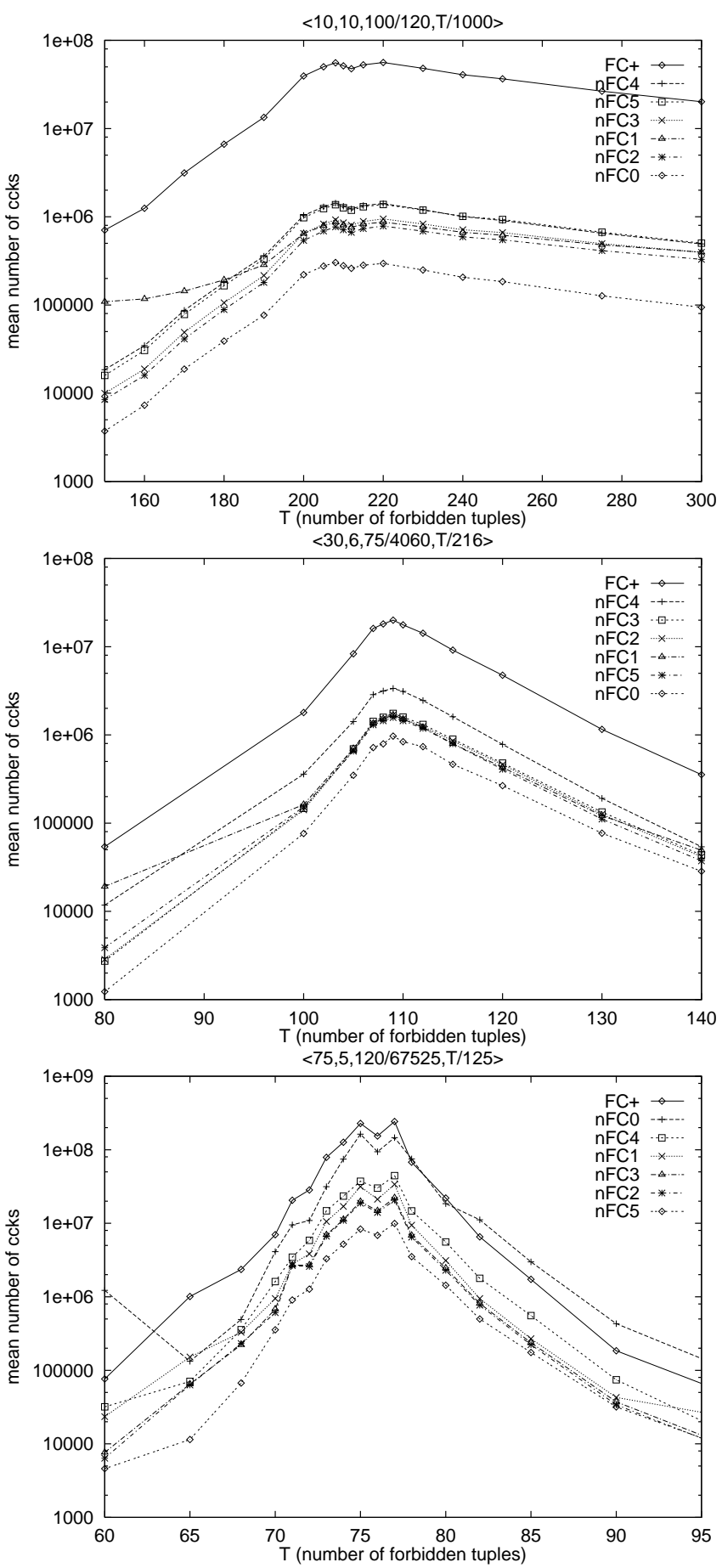

Fig. 3. Average number of checks for three classes of ternary random problems. 

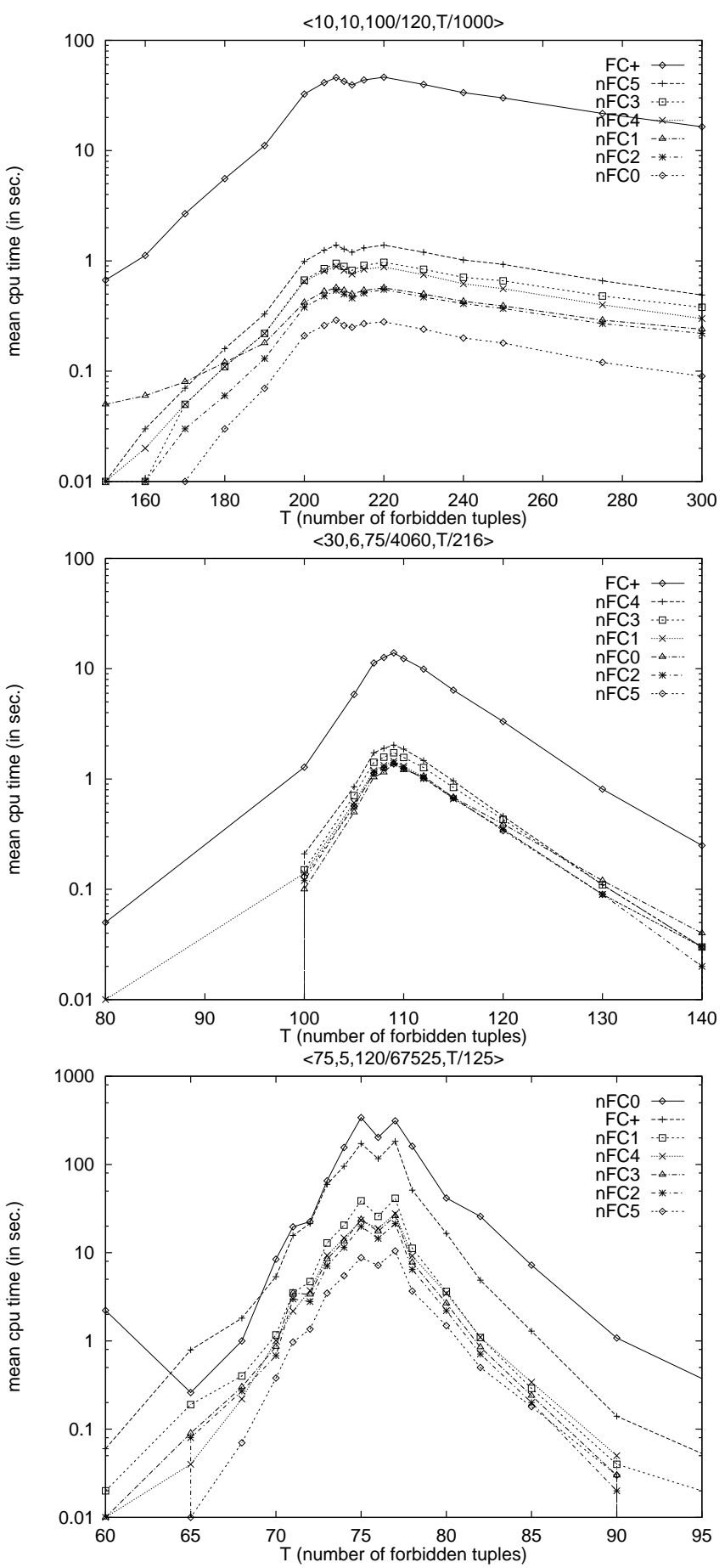

Fig. 4. Average cpu time for three classes of ternary random problems. 
algorithms $\mathrm{nFC} 1$ to $\mathrm{nFC} 5$ clearly outperform $\mathrm{nFC} 0$. Even $\mathrm{FC}+$ performs better than $\mathrm{nFC} 0$. The winner is $\mathrm{nFC} 5$, the algorithm which performs the greatest effort per node, and causes the highest filtering. It is 32 times faster than $\mathrm{nFC} 0$ at the peak. The good behaviour of $\mathrm{nFC} 4$ in number of visited nodes has no translation in performance. However, $\mathrm{nFC} 2$ visiting more nodes than $\mathrm{nFC} 4$ is the second best algorithm in performance in the three problem classes. Considering $\mathrm{FC}+$, it has the worst performance for loose and medium constraints, and it is the second worst (after nFC0) for tight constraints. Any of the proposed algorithms outperforms $\mathrm{FC}+$ in the three problem classes.

We can also point out some other noteworthy phenomena that are not visible in the figures reported here. First, on the problem classes presented there, $\mathrm{nFC0}$ is the only algorithm that encountered an exceptionally hard problem, located in the satisfiable region of the $\left\langle 75,5,120 / 67525, p_{2}\right\rangle$ class. Second, when the heuristic minimum domain size for variable selection is used instead of minimum $\frac{\text { domain size }}{\text { degree }}, \mathrm{nFC} 0$ becomes more frequently subject to thrashing, even on problem sizes remaining very easy for the algorithms $\mathrm{nFC} 1$ to $\mathrm{nFC5}$.

\section{Summary and Conclusion}

We presented several possible generalizations of the FC algorithm to non-binary constraint networks. We studied their properties, and analyzed their complexities. We also compared these non-binary algorithms to the binary FC + algorithm, which runs on the hidden conversion of non-binary networks.

We provided initial empirical results on the performance of these algorithms. From them, we conclude that the proposed algorithms outperform existing approaches on sparse problems with tight constraints. On easier problems, the benefits caused by their early lookahead do not outweigh the propagation effort. This unsurprising conclusion fits the already known trade-off between benefits and costs in constraint satisfaction. Nevertheless, more empirical studies are needed to substantiate which of these algorithms are promising, and on which constraints they perform better. An ultimate goal could be to exhibit a criterion under which to decide when a constraint should be processed by the $\mathrm{nFC} 0$ principle, and when it should be propagated with a more pruningful mechanism. The result would be a mixed algorithm, taking the best of both techniques.

\section{References}

1. F. Bacchus and P. van Beek. On the conversion between non-binary and binary constraint satisfaction problems. In Proceedings AAAI'98, pages 311-318, Madison WI, 1998.

2. C. Bessière and J.C. Régin. Arc consistency for general constraint networks: preliminary results. In Proceedings IJCAI'97, pages 398-404, Nagoya, Japan, 1997.

3. R. Dechter. On the expressiveness of networks with hidden variables. In Proceedings AAAI'90, pages 556-562, Boston MA, 1990.

4. R.M. Haralick and G.L. Elliot. Increasing tree seach efficiency for constraint satisfaction problems. Artificial Intelligence, 14:263-313, 1980. 
5. J. Larrosa and P. Meseguer. Adding constraint projections in n-ary csp. In J.C. Régin and W. Nuijtens, editors, Proceedings of the ECAI'98 workshop on nonbinary constraints, pages 41-48, Brighton, UK, 1998.

6. A.K. Mackworth. On reading sketch maps. In Proceedings IJCAI'r7, pages 598606, Cambridge MA, 1977.

7. R. Mohr and G. Masini. Good old discrete relaxation. In Proceedings ECAI'88, pages 651-656, Munchen, FRG, 1988.

8. F. Rossi, C. Petrie, and V. Dhar. On the equivalence of constraint satisfaction problems. In Proceedings ECAI'90, pages 550-556, Stockholm, Sweden, 1990.

9. B. Smith. Phase transition and the mushy region in constraint satisfaction problems. In Proceedings ECAI'94, pages 100-104, Amsterdam, The Netherlands, 1994.

10. Pascal Van Hentenryck. Constraint Satisfaction in Logic Programming. MIT Press, Cambridge, MA, 1989. 